\title{
On a difficulty in the formulation of initial and boundary conditions for eigenfunction expansion solutions for the start-up of fluid flow
}

\author{
Ivan C. Christov
}

Department of Mechanical and Aerospace Engineering, Princeton University, Princeton, NJ 08544, USA

\begin{abstract}
Most mathematics and engineering textbooks describe the process of "subtracting off" the steady state of a linear parabolic partial differential equation as a technique for obtaining a boundary-value problem with homogeneous boundary conditions that can be 'solved by separation of variables (i.e., eigenfunction expansions). While this method produces the correct solution for the start-up of the flow of, e.g., a Newtonian fluid between parallel plates, it can lead to erroneous solutions to the corresponding problem for a class of non-Newtonian fluids. We show that the reason for this is the non-rigorous enforcement of the start-up condition in the textbook approach, which leads to a violation of the principle of causality. Nevertheless, these boundary-value problems can be solved correctly using eigenfunction expansions, and we present the formulation that makes this possible (in essence, an application of Duhamel's principle). The solutions obtained by this new approach are shown to agree identically with those obtained by using the Laplace transform in time only, a technique that enforces the proper start-up condition implicitly (hence, the same error cannot be committed).
\end{abstract}

Keywords: start-up flows, Stokes' first problem, eigenfunction expansions, Jeffreys fluid, Laplace transform, Duhamel's principle

\section{Introduction: Stokes' first problem on a strip (start-up of plane Couette flow)}

Suppose an incompressible Newtonian fluid with kinematic viscosity $v$ fills the gap between two parallel horizontal plates a distance $d$ apart. We are interested in the problem of the bottom plate suddenly being set into motion at $t=0^{+}$with constant velocity $U_{0}$ in the positive $x$ direction. Known as plane Couette flow, this is a variation on a problem first considered by Sir George Gabriel Stokes (Stokes, 1851), nowadays called "Stokes' first problem," which has applications in rheometry and creep tests of deformation (Nijenhuis et al., 2007). Stokes-type problems remain an important aspect of modern non-Newtonian fluid mechanics (Duarte et al., 2008; Pritchard et al., 2011; McArdle et al., 2012; Vasquez et al., 2013). Choosing the dimensionless variables $u=u^{\star} / U_{0}, y=y^{\star} / d$ and $t=t^{\star} /\left(d^{2} / v\right)$, we must solve an initial-boundary-value problem (IBVP) for the $x$-component of the velocity field, $u=u(y, t)$, of the fluid (see, e.g., Batchelor, 1967; Leal, 2007):

$$
\begin{aligned}
\frac{\partial u}{\partial t} & =\frac{\partial^{2} u}{\partial y^{2}}, & & \quad(y, t) \in(0,1) \times(0, \infty), \\
u(y, 0) & =0, & & 0<y<1, \\
u(0, t) & =1, & & t>0, \\
u(1, t) & =0, & & t>0 .
\end{aligned}
$$

Email address: christov@alum.mit.edu (Ivan C. Christov) URL: http://alum.mit.edu/www/christov (Ivan C. Christov)
In what follow we shall contrast the latter IBVP with an example IBVP corresponding to the plane Couette flow of an incompressible rate-type non-Newtonian fluid, the rheology of which we take to have the dimensionless form

$$
\sigma+\tau \frac{\partial \sigma}{\partial t}=\dot{\gamma}+\alpha \frac{\partial \dot{\gamma}}{\partial t}
$$

where $\sigma$ is the shear stress, $\dot{\gamma}=\partial u / \partial y$ is the rate of strain, $\tau=\lambda_{1} /\left(d^{2} / v\right)$ is the dimensionless relaxation time, and $\alpha=$ $\lambda_{2} /\left(d^{2} / v\right)$ is the dimensionless retardation time with $0<\lambda_{2}<$ $\lambda_{1}$ in order to have a causal and well-posed model (see, e.g., Bird et al., 1987, Sect. 5.2b). Now, eliminating $\sigma$ between Eq. (2) and Cauchy's first law $\partial u / \partial t=\partial \sigma / \partial y$ (see, e.g., (Tanner, 1962; Christov, 2010)), the non-Newtonian version of IBVP (1) that we shall consider takes the form

$$
\begin{aligned}
\left(1+\tau \frac{\partial}{\partial t}\right) \frac{\partial u}{\partial t} & =\frac{\partial}{\partial y}\left(1+\alpha \frac{\partial}{\partial t}\right) \frac{\partial u}{\partial y}, \quad(y, t) \in(0,1) \times(0, \infty) \\
u(y, 0) & =\frac{\partial u}{\partial t}(y, 0)=0, \quad 0<y<1, \\
u(0, t) & =1, \quad t>0, \quad t>0 . \\
u(1, t) & =0, \quad 3 \mathrm{~b})
\end{aligned}
$$

To the best of our knowledge, the correct solution to IBVP (3) cannot be found in the literature.

Equation (2) is attributed (Joseph, 1986; Bird et al., 1987) to Sir Harold Jeffreys (Jeffreys, 1929, 1932), and it appears to have been independently proposed as a constitutive relation for polymeric suspensions (Frohlich and Sack, 1946). 
This model has been successfully applied to experimental data (Toms and Strawbridge, 1953), it has been generalized in a frame-indifferent manner to multiple dimensions by Oldroyd (1950), and more recently it was extended to apply to bubbly liquids as well (Llewellin et al., 2002). Jeffreys' rheology 1 covers the well-known relaxation model due to James Clerk Maxwell (Maxwell, 1867) as special case in the limit of $\alpha \rightarrow 0$. Meanwhile the rheology of the so-called second-order (or second-grade) fluid (Coleman and Noll, 1960, Sect. 7) undergoing planar unidirectional flows is recovered in the limit of $\tau \rightarrow 0$. Thus, for our purposes, IBVP (3) is a sufficiently general non-Newtonian start-up problem. Finally, note that Eq. 3a is also a generic model for the evolution of linearly dissipativedispersive wave packets (Christov, 2008).

Though it is standard practice (Carslaw and Jaeger, 1959; Batchelor, 1967; Leal, 2007) to write the boundary conditions as done in Eqs. (1c) and (3c), note that this leaves a piece of the problem somewhat vague, i.e., not explicitly stated. That is, prior to start-up (i.e., for $t<0) u \equiv 0$. This means that the boundary condition for Stokes' first problem suffers a jump discontinuity across the plane $t=0$. Hence, the mathematicallycorrect way of writing it is $u(0, t)=H(t)$. The purpose of the present article is to show that this is not merely a semantic distinction of no consequence, and that it fundamentally affects the method of solution. Specifically, one cannot arrive at the correct solution of IBVP (3) using the boundary condition $u(0, t)=1$ together with the textbook techniques (Carslaw and Jaeger, 1959; Batchelor, 1967; Leal, 2007; Bruus, 2008). Meanwhile, the solution to IBVP (1) happens to be unaffected. This has significant implications not only for problems of viscoelastic fluid flow (as in the featured example) but also for problems of hyperbolic, delayed or otherwise nonclassical heat conduction, wherein similar IBVPs arise (Tzou, 1997; Straughan, 2011). Such IBVPs are also relevant in acoustics, where they are referred to as signaling problems (Crighton, 1998). Historically, identifying such apparent mathematical "difficulties" has proven worthwhile, e.g., the theory of shock waves was developed, in part, because of Stokes' paper "On a difficulty in the theory of sound" (Stokes, 1848).

This paper is organized as follows. In Sect.2, the solutions of IBVPs (1) and (3) are found by the textbook eigenfunction expansion technique. In Sect. 3, the solutions are derived by using only the Laplace transform in time, showing that the Laplacetransform solution to IBVP (3) does not agree with the solution found in Sect. 2. Then, in Sect. 4, the textbook eigenfunction expansion technique is modified to satisfy causality and shown to reproduce identically the (correct) solutions to both IBVPs found in Sect. 3. Sect. 5 gives a critical discussion of the literature on start-up flows in the context of the present work.

\footnotetext{
${ }^{1}$ Though many recent papers refer to Eq. 3a as corresponding to an "Oldroyd-B fluid," it should be noted that the contribution of Oldroyd is in the nonlinear terms of the rheological model, which are self-canceling for planar unidirectional flows. Therefore, there is no sense in which to distinguish between Oldroyd's upper-convected ("B") and lower-convected ("A") time rates, and it is more appropriate to credit this equation to Jeffreys, as done in the textbook of Bird et al. (1987).
}

\section{Solution by separation of variables through a transfor- mation to homogeneous boundary conditions}

If we assume $u$ is independent of $t$, then we obtain the steadystate solution of both IBVPs (1) and (3), namely

$$
u_{\mathrm{ss}}(y)=1-y .
$$

Using this observation, the textbook approach (Carslaw and Jaeger, 1959; Batchelor, 1967; Leal, 2007; Bruus, 2008) is to now make the change of dependent variable

$$
u(y, t)=v(y, t)+u_{\mathrm{ss}}(y)
$$

where $v(0, t)=v(1, t)=0$, unlike $u(y, t)$. Noting that $\partial u_{\mathrm{ss}} / \partial t=$ $\partial^{2} u_{\mathrm{ss}} / \partial y^{2}=0$, IBVP (1) becomes

$$
\begin{aligned}
\frac{\partial v}{\partial t} & =\frac{\partial^{2} v}{\partial y^{2}}, \quad(y, t) \in(0,1) \times(0, \infty), \\
v(y, 0) & =-u_{\mathrm{ss}}(y), \quad 0<y<1, \\
v(0, t) & =0, \quad t>0, \\
v(1, t) & =0, \quad t>0 .
\end{aligned}
$$

Similarly, IBVP (3) becomes

$$
\begin{aligned}
\left(1+\tau \frac{\partial}{\partial t}\right) \frac{\partial v}{\partial t} & =\frac{\partial}{\partial y}\left(1+\alpha \frac{\partial}{\partial t}\right) \frac{\partial v}{\partial y}, \quad(y, t) \in(0,1) \times(0, \infty) \\
v(y, 0) & =-u_{\mathrm{ss}}(y), \quad \frac{\partial v}{\partial t}(y, 0)=0, \quad 0<y<1, \quad(7 \mathrm{~b}) \\
v(0, t) & =0, \quad t>0, \\
v(1, t) & =0, \quad t>0 .
\end{aligned}
$$

It is well known (Titchmarsh, 1962) that the eigenvalue problem

$$
\frac{\mathrm{d}^{2}}{\mathrm{~d} y^{2}} \psi_{n}(y)=-\lambda_{n} \psi_{n}(y), \quad \psi_{n}(0)=\psi_{n}(1)=0
$$

possesses a complete set of orthogonal eigenfunctions $\left\{\psi_{n}(y)\right\}_{n=1}^{\infty}$, namely

$$
\begin{aligned}
\psi_{n}(y)=\sin \left(\sqrt{\lambda_{n}} y\right), \quad \lambda_{n} & =n^{2} \pi^{2}, \\
& \int_{0}^{1} \psi_{n}(y) \psi_{m}(y) \mathrm{d} y=\frac{1}{2} \delta_{n m},
\end{aligned}
$$

where $\delta_{n m}$ is the Kronecker delta symbol and $n, m=1,2, \ldots$.

With this in mind, the method of separation of variables (see, e.g., Sneddon, 2006, Chap. 6, Sect. 4) suggests the ansatz $v(y, t)=\sum_{n} a_{n}(t) \psi_{n}(y)$. Substituting the latter into Eq. 6a and using the orthogonality relation from Eq. (9), we see that $a_{n}$ must satisfy

$$
\frac{\mathrm{d} a_{n}}{\mathrm{~d} t}=-\lambda_{n} a_{n}
$$

whence $a_{n}(t)=a_{n}(0) \mathrm{e}^{-\lambda_{n} t}$ and $a_{n}(0)=2 \int_{0}^{1} v(y, 0) \psi_{n}(y) \mathrm{d} y=$ $-2 /(n \pi)$. Thus, we would be led to believe that the solution to IBVP (1) is

$$
u(y, t)=(1-y)-\frac{2}{\pi} \sum_{n=1}^{\infty} \exp \left(-n^{2} \pi^{2} t\right) \frac{\sin (n \pi y)}{n} .
$$


Indeed this is precisely the dimensionless version of the expression found in Eq. (1) (with $v_{1}=1, v_{2}=0$ and $f(x)=$ 0 ) in Sect. 3.4 of the book of Carslaw and Jaeger (1959), in Eq. (4.3.14) in Sect. 4.3 of the book of Batchelor (1967) and in Eq. (3-158) in Chap. 3F of the book of Leal (2007). However, notice that (due to our choice in notation) we must keep in mind that this solution does not apply for $t<0$ because prior to start-up $u \equiv 0$, which is not true for the expression in Eq. 111.

Similarly, for the Jeffreys fluid, by substituting the ansatz $v(y, t)=\sum_{n} a_{n}(t) \psi_{n}(y)$ into Eq. (7a) and using the orthogonality relation from Eq. (9), we see that $a_{n}$ must satisfy

$$
\left(1+\tau \frac{\mathrm{d}}{\mathrm{d} t}\right) \frac{\mathrm{d} a_{n}}{\mathrm{~d} t}=-\lambda_{n}\left(1+\alpha \frac{\mathrm{d}}{\mathrm{d} t}\right) a_{n}
$$

The characteristic polynomial of this ordinary differential equation (ODE) is $\tau r^{2}+\left(1+\lambda_{n} \alpha\right) r+\lambda_{n}$; its roots are $r_{ \pm}=$ $-\left[\left(1+\lambda_{n} \alpha\right) \pm \sqrt{\Delta_{n}}\right] /(2 \tau)$, where $\Delta_{n}:=\left(1+\lambda_{n} \alpha\right)^{2}-4 \lambda_{n} \tau$. Thus, three cases can be distinguished based on the sign of $\Delta_{n}$, and we arrive at

$$
\begin{aligned}
& a_{n}(t)=\exp \left(-\frac{1+\lambda_{n} \alpha}{2 \tau} t\right) \\
& \quad \times \begin{cases}\mathfrak{c}_{n} \sinh \left(\frac{t}{2 \tau} \sqrt{\Delta_{n}}\right)+\mathfrak{d}_{n} \cosh \left(\frac{t}{2 \tau} \sqrt{\Delta_{n}}\right), & \Delta_{n}>0, \\
\mathfrak{c}_{n} t+\mathfrak{d}_{n} & \Delta_{n}=0, \\
\mathfrak{c}_{n} \sin \left(\frac{t}{2 \tau} \sqrt{\left|\Delta_{n}\right|}\right)+\mathfrak{d}_{n} \cos \left(\frac{t}{2 \tau} \sqrt{\left|\Delta_{n}\right|}\right), & \Delta_{n}<0,\end{cases}
\end{aligned}
$$

where the constants $\mathfrak{c}_{n}$ and $\mathfrak{d}_{n}$ are determined from the initial conditions in Eq. (7b) as follows

$$
\begin{aligned}
&-\frac{2}{n \pi}=a_{n}(0)=\mathfrak{d}_{n}, \\
& 0=\frac{\mathrm{d} a_{n}}{\mathrm{~d} t}(0)= \begin{cases}-\frac{1+\lambda_{n} \alpha}{2 \tau} \mathfrak{d}_{n}+\frac{\sqrt{\left|\Delta_{n}\right|}}{2 \tau} \mathfrak{c}_{n}, & \Delta_{n} \neq 0, \\
-\frac{1+\lambda_{n} \alpha}{2 \tau} \mathfrak{d}_{n}+\mathfrak{c}_{n}, & \Delta_{n}=0 .\end{cases}
\end{aligned}
$$

Thus, we would be led to believe that the solution to IBVP (3) is

$$
u(y, t)=(1-y)-\frac{2}{\pi} \sum_{n=1}^{\infty} \exp \left(-\frac{1+\lambda_{n} \alpha}{2 \tau} t\right) A_{n}(t) \frac{\sin (n \pi y)}{n}
$$

where

$$
A_{n}(t)= \begin{cases}\frac{1+\lambda_{n} \alpha}{\sqrt{\Delta_{n}}} \sinh \left(\frac{t}{2 \tau} \sqrt{\Delta_{n}}\right)+\cosh \left(\frac{t}{2 \tau} \sqrt{\Delta_{n}}\right), & \Delta_{n}>0, \\ \frac{1+\lambda_{n} \alpha}{2 \tau} t+1, & \Delta_{n}=0, \\ \frac{1+\lambda_{n} \alpha}{\sqrt{\left|\Delta_{n}\right|}} \sin \left(\frac{t}{2 \tau} \sqrt{\left|\Delta_{n}\right|}\right)+\cos \left(\frac{t}{2 \tau} \sqrt{\left|\Delta_{n}\right|}\right), & \Delta_{n}<0 .\end{cases}
$$

Again, due to our choice in notation, we must keep in mind that this solution does not apply for $t<0$.

Let us now find the start-up solutions by another analytical technique. If we have not made any mistakes so far, then the existence and uniqueness theorems for linear BVPs (Courant and Hilbert, 1962) guarantee that any solutions found by a different technique must agree with the solutions from this section.

\section{Solution using only the Laplace transform in time}

An alternative solution technique is to apply the Laplace transform in time (see, e.g., the classic comprehensive treatment by Doetsch (1974)), namely

$$
\mathcal{L}\{u(y, t)\}:=\int_{0}^{\infty} \mathrm{e}^{-s t} u(y, t) \mathrm{d} t \equiv \bar{u}(y, s), \quad s \in \mathbb{C} .
$$

Now, the condition of start-up, i.e., $u(0, t) \equiv 0$ for $t<0$ is implicit because the Laplace transform is only well defined for distributions whose action on tests functions with support in $t \in(-\infty, 0)$ vanishes identically (Doetsch, 1974, Sect. 12). (This is related to the principle of causality (Toll, 1956), i.e., the Laplace transform is a causal operator only for $u(\cdot, t)$ such that $u(\cdot, t) \equiv 0$ for $t<0$.) As a consequence, $\mathcal{L}\{1\}$ must be understood in the sense $\mathcal{L}\{H(t)\}$, where $\mathcal{L}\{H(t)\}=1 / s$, while initial conditions imposed on $u$ must be understood in the sense of $\lim _{t \rightarrow 0^{-}} u(y, t)$ because $\lim _{t \rightarrow 0^{+}} u(y, t)$ does not have to attain this same value (see Doetsch (1974, pp. 70-71, 129) and Miller and Mattuck (2010)).

Applying the Laplace transform to IBVP (1) gives the $s u b$ sidiary boundary value problem

$$
\begin{aligned}
s \bar{u} & =\frac{\partial^{2} \bar{u}}{\partial y^{2}}, \quad y \in(0,1), \\
\bar{u}(0, s) & =\frac{1}{s} \\
\bar{u}(1, s) & =0 .
\end{aligned}
$$

The solution of the latter BVP is easily found to be

$$
\bar{u}(y, s)=\frac{\sinh [\sqrt{s}(1-y)]}{s \sinh (\sqrt{s})} .
$$

Noting that the poles (in the complex plane) of the right-hand side are at $s$ such that $s=0$ or $\sqrt{s}=\mathrm{i} n \pi, n=1,2, \ldots$, it is a standard exercise (see, e.g., Carslaw and Jaeger, 1963, Sect. 40) to evaluate the Laplace inversion integral using a Bromwichtype contour in the complex plane and the residue theorem, arriving at

$$
u(y, t)=H(t)\left[(1-y)-\frac{2}{\pi} \sum_{n=1}^{\infty} \exp \left(-n^{2} \pi^{2} t\right) \frac{\sin (n \pi y)}{n}\right] .
$$

Note the steady state is, as expected, $u_{\mathrm{ss}}(y) \equiv \lim _{t \rightarrow \infty} u(y, t)=$ $1-y$ but the change of variables given in Eq. (5) does not eliminate this term from the solution in Eq. (20) because this change of variables does not leave the solution identically equal to zero for $t<0$, as required by the condition of start-up. We shall further elaborate on this point below. 
Similarly, IBVP (3) can be transformed into

$$
\begin{aligned}
\frac{s(1+\tau s)}{1+\alpha s} \bar{u} & =\frac{\partial^{2} \bar{u}}{\partial y^{2}}, \quad y \in(0,1), \\
\bar{u}(0, s) & =\frac{1}{s}, \\
\bar{u}(1, s) & =0 .
\end{aligned}
$$

The solution of the latter BVP is easily found to be

$$
\bar{u}(y, s)=\frac{\sinh [\sqrt{\zeta(s)}(1-y)]}{s \sinh [\sqrt{\zeta(s)}]}, \quad \zeta(s):=\frac{s(1+\tau s)}{1+\alpha s} .
$$

The poles of $\bar{u}$ are at $s$ such that $\sqrt{\zeta(s)}=$ in $\pi$, i.e., at $s=$ $\left\{0,-\tau^{-1}, s_{n}^{ \pm}\right\}$, where

$$
s_{n}^{ \pm}=\frac{-\left(1+n^{2} \pi^{2} \alpha\right) \pm \sqrt{\left(1+n^{2} \pi^{2} \alpha\right)^{2}-4 \tau n^{2} \pi^{2}}}{2 \tau}, \quad n=1,2, \ldots
$$

As before, we can use the Laplace inversion formula with a Bromwich-type contour, and all that is left to do is find the residues of $\mathrm{e}^{s t} \bar{u}(s, t)$ at the poles (see Carslaw and Jaeger, 1963))

The pole at $s=0$ is of order 2 because both $s$ and $\sinh [\sqrt{\zeta(s)}]$ vanish, hence the residue is given by

$$
\lim _{s \rightarrow 0} \frac{\mathrm{d}}{\mathrm{d} s}\left\{s^{2} \frac{\mathrm{e}^{s t} \sinh [\sqrt{\zeta(s)}(1-y)]}{s \sinh [\sqrt{\zeta(s)}]}\right\}=1-y .
$$

The pole at $s=-\tau^{-1}$ is simple and its residue is easily found to be zero. Then, since $s_{n}^{ \pm}=\left[-\left(1+n^{2} \pi^{2} \alpha\right) \pm \sqrt{\Delta_{n}}\right] /(2 \tau)$, where $\Delta_{n}=\left(1+n^{2} \pi^{2} \alpha\right)^{2}-4 \tau n^{2} \pi^{2}$, the poles at $s=s_{n}^{ \pm}$for the case $\Delta_{n} \neq 0$ are simple and the corresponding residues are given by

$$
\frac{\mathrm{e}^{s_{n}^{ \pm} t} \sinh [\mathrm{i} n \pi(1-y)]}{\left.\frac{\mathrm{d}}{\mathrm{d} s}(s \sinh [\sqrt{\zeta(s)}])\right|_{s=s_{n}^{ \pm}}}=\frac{\mathrm{e}^{s_{n}^{ \pm} t} \sinh [\mathrm{i} n \pi(1-y)]}{\left.s \cosh [\sqrt{\zeta(s)}] \frac{\zeta^{\prime}(s)}{2 \sqrt{\zeta(s)}}\right|_{s=s_{n}^{ \pm}} .}
$$

Note that $\sinh [i n \pi(1-y)] / \cosh (i n \pi)=-\mathrm{i} \sin (n \pi y)$ and

$$
\left\{\left.s \frac{\zeta^{\prime}(s)}{2 \sqrt{\zeta(s)}}\right|_{s=s_{n}^{ \pm}}\right\}^{-1}=\frac{\mp 1 \pm n^{2} \pi^{2} \alpha+\sqrt{\Delta_{n}}}{\mathrm{i} n \pi \sqrt{\Delta_{n}}} .
$$

Hence, the expression on the right-hand side of Eq. 25 equals

$$
-\frac{\sin (n \pi y)}{n \pi} \mathrm{e}^{s_{n}^{ \pm} t}\left(\frac{ \pm 1 \mp n^{2} \pi^{2} \alpha}{\sqrt{\Delta_{n}}}+1\right) .
$$

Since Eq. 27 corresponds, for a given $n$, to the residues at two distinct poles, and eventually we have so add-up all residues,

\footnotetext{
${ }^{2}$ For these calculations the reader is encouraged to make use of a computer algebra system such as the software package MathEMATICA.
}

we can sum these now to obtain

$$
\begin{aligned}
& -\frac{2 \sin (n \pi y)}{n \pi} \exp \left(-\frac{1+n^{2} \pi^{2} \alpha}{2 \tau} t\right) \\
& \times \begin{cases}\frac{1-n^{2} \pi^{2} \alpha}{\sqrt{\Delta_{n}}} \sinh \left(\frac{t}{2 \tau} \sqrt{\Delta_{n}}\right)+\cosh \left(\frac{t}{2 \tau} \sqrt{\Delta_{n}}\right), & \Delta_{n}>0, \\
\frac{1-n^{2} \pi^{2} \alpha}{\sqrt{\left|\Delta_{n}\right|}} \sin \left(\frac{t}{2 \tau} \sqrt{\left|\Delta_{n}\right|}\right)+\cos \left(\frac{t}{2 \tau} \sqrt{\left|\Delta_{n}\right|}\right), & \Delta_{n}<0 .\end{cases}
\end{aligned}
$$

Finally, for the case of $\Delta_{n}=0$, we note that the limit $\Delta_{n} \rightarrow 0$ is well-defined from both above and below because taking it in either expression in Eq. 28) yields

$$
-\frac{2 \sin (n \pi y)}{n \pi} \exp \left(-\frac{1+n^{2} \pi^{2} \alpha}{2 \tau} t\right)\left(\frac{1-n^{2} \pi^{2} \alpha}{2 \tau} t+1\right),
$$

hence this is the residue for $\Delta_{n}=0$.

Setting $\lambda_{n}=n^{2} \pi^{2}$, adding the expressions in Eqs. (24), (28) and (29) together, where the last two must also be summed over all allowed $n$, and premultiplying by $H(t)$, we obtain the solution to IBVP (3):

$u(y, t)=H(t)\left[(1-y)-\frac{2}{\pi} \sum_{n=1}^{\infty} \exp \left(-\frac{1+\lambda_{n} \alpha}{2 \tau} t\right) A_{n}(t) \frac{\sin (n \pi y)}{n}\right]$,

where

$$
A_{n}(t)= \begin{cases}\frac{1-\lambda_{n} \alpha}{\sqrt{\Delta_{n}}} \sinh \left(\frac{t}{2 \tau} \sqrt{\Delta_{n}}\right)+\cosh \left(\frac{t}{2 \tau} \sqrt{\Delta_{n}}\right), & \Delta_{n}>0, \\ \frac{1-\lambda_{n} \alpha}{2 \tau} t+1, & \Delta_{n}=0, \\ \frac{1-\lambda_{n} \alpha}{\sqrt{\left|\Delta_{n}\right|}} \sin \left(\frac{t}{2 \tau} \sqrt{\left|\Delta_{n}\right|}\right)+\cos \left(\frac{t}{2 \tau} \sqrt{\left|\Delta_{n}\right|}\right), & \Delta_{n}<0 .\end{cases}
$$

Evidently the only difference between Eqs. (16) and (31) is that in the pre-factors of the sinh, $t$ and $\sin$ terms $+\lambda_{n} \alpha$ has been changed to $-\lambda_{n} \alpha$.

Let us now try to resolve the apparent contradiction that Eqs. (15)-16) and Eqs. (30-31), which are not identical, were both found to be solutions to the same linear IBVP.

\section{Resolution to the apparent difficulty, or how to find the correct solution using an eigenfunction expansion}

It should be obvious that Eqs. (11) and (15) are not mathematically equivalent to Eqs. (20) and (30) due to "implicit assumptions" about start-up. Only Eqs. (20) and (30) account for all the stipulations of the start-up problem in a self-contained mathematical fashion. Thus, guided by these expressions, we modify the transformation in Eq. (5) as follows:

$$
v(y, t)=u(y, t)-H(t) u_{\mathrm{ss}}(y) .
$$

Of course, one may argue this is merely a semantic point because it is understood that $u_{\mathrm{ss}}(y)$ in Eq. (5) only "makes sense" 
for $t>0$. However, because the governing PDEs contain partial time derivatives, the transformations in Eqs. (5) and (32) are not equivalent. Thus, it is important that the start-up condition is always written explicitly (though the appropriate $H(t)$ prefactor) to obtain the physical solution to the start-up problem and, moreover, to not alter the state of rest prior to start-up.

Noting that $\partial\left(H(t) u_{\mathrm{ss}}(y)\right) / \partial t=\delta(t) u_{\mathrm{ss}}(y)$, where $\delta(t)$ is the Dirac delta distribution, and $\partial^{2}\left(H(t) u_{\mathrm{ss}}(y)\right) / \partial y^{2}=0$, IBVP (1) becomes

$$
\begin{aligned}
\frac{\partial v}{\partial t} & =\frac{\partial^{2} v}{\partial y^{2}}-\delta(t) u_{\mathrm{ss}}(y), \quad(y, t) \in(0,1) \times(0, \infty), \\
v(y, 0) & =0, \quad 0<y<1, \\
v(0, t) & =0, \quad t>0, \\
v(1, t) & =0, \quad t>0 .
\end{aligned}
$$

The initial condition, being understood as the state prior to startup, i.e., as $\lim _{t \rightarrow 0^{-}} u(y, t)$, naturally remains zero. This interpretation is a demonstration of Duhamel's principle (Duhamel, 1833; Bartels and Churchill, 1942; Sneddon, 2006), namely, that a time-varying boundary condition can be "exchanged" for a homogeneous boundary condition at the "cost" of adding a time-varying source term to the linear BVP. Notice that the textbook approach exchanges the inhomogeneous boundary conditions for a homogeneous boundary condition at the cost of an inhomogeneous initial condition. Philosophically, this is already problematic because the cumulative effects of the boundary condition from $t=0$ up to $t=\infty$ have been "condensed" into an initial condition and imposed $t=0$, an act that readily violates the principle of causality, namely "no output before the input" (Toll, 1956) 3

As before, the method of separation of variables suggests the ansatz $v(y, t)=\sum_{n} a_{n}(t) \psi_{n}(y)$. Substituting the latter into Eq. (33a) and using the orthogonality relation from Eq. (9), we see that $a_{n}$ must satisfy

$$
\frac{\mathrm{d} a_{n}}{\mathrm{~d} t}=-\lambda_{n} a_{n}-\frac{2}{n \pi} \delta(t), \quad a_{n}(0)=0 .
$$

Taking the Laplace transform, we obtain $\left(s+\lambda_{n}\right) \bar{a}_{n}=-2 /(n \pi)$, and it follows that $a_{n}(t)=-2 /(n \pi) H(t) \mathrm{e}^{-\lambda_{n} t} 4$ Thus, the complete solution to IBVP (1) is precisely

$$
u(y, t)=H(t)\left[(1-y)-\frac{2}{\pi} \sum_{n=1}^{\infty} \exp \left(-n^{2} \pi^{2} t\right) \frac{\sin (n \pi y)}{n}\right],
$$

which is identical to the Laplace-transform-in-time-only solution given in Eq. (20). Unlike the expression in Eq. (11), the solution in Eq. (35) is valid for all $t \in \mathbb{R}$, identically satisfying the condition that the fluid is at rest prior to $t=0$.

${ }^{3}$ It should be noted that there are deeper issues regarding causality in relation to viscous (compressible) flow that are beyond the scope of the present work (Jordan et al, 2000).

${ }^{4}$ Notice that the Laplace domain solution is identical to the corresponding one for the ODE in Eq. 10, where the $-2 /(n \pi)$ term is contributed by the initial condition $a_{n}(0)=-2 /(n \pi)$.
Similarly, IBVP (3) becomes

$$
\begin{aligned}
\left(1+\tau \frac{\partial}{\partial t}\right) \frac{\partial v}{\partial t}=\frac{\partial}{\partial y}\left(1+\alpha \frac{\partial}{\partial t}\right) \frac{\partial v}{\partial y}-\left[\delta(t)+\tau \delta^{\prime}(t)\right] u_{\mathrm{ss}}(y) & \\
v(y, 0) & =\frac{\partial v}{\partial y}(y, 0)=0, \quad 0<y<1 \\
v(0, t) & =0, \quad t>0 \\
v(1, t) & =0, \quad t>0
\end{aligned}
$$

Once again we note the effect of Duhamel's principle: the inhomogeneous boundary condition was exchanged for a source term. Substituting the separation of variables ansatz into Eq. (36a) and using the orthogonality relation from Eq. (9), we find that $a_{n}$ satisfies

$$
\begin{array}{r}
\left(1+\tau \frac{\mathrm{d}}{\mathrm{d} t}\right) \frac{\mathrm{d} a_{n}}{\mathrm{~d} t}=-\lambda_{n}\left(1+\alpha \frac{\mathrm{d}}{\mathrm{d} t}\right) a_{n}-\frac{2}{n \pi}\left[\delta(t)+\tau \delta^{\prime}(t)\right], \\
a_{n}(0)=\frac{\mathrm{d} a_{n}}{\mathrm{~d} t}(0)=0 .
\end{array}
$$

Taking the Laplace transform of the latter, we obtain

$$
(1+\tau s) s \bar{a}_{n}=-\lambda_{n}(1+\alpha s) \bar{a}_{n}-\frac{2}{n \pi}(1+\tau s) .
$$

Meanwhile, if we take the Laplace transform of Eq. (12), we find that

$$
(1+\tau s) s \bar{a}_{n}-(1+\tau s) a_{n}(0)-\tau \frac{\mathrm{d} a_{n}}{\mathrm{~d} t}(0)=-\lambda_{n}(1+\alpha s) \bar{a}_{n}+\lambda_{n} \alpha a_{n}(0),
$$

which, after imposing the initial conditions from Eq. (14), becomes

$$
(1+\tau s) s \bar{a}_{n}=-\lambda_{n}(1+\alpha s) \bar{a}_{n}-\frac{2}{n \pi}\left(1+\tau s+\lambda_{n} \alpha\right) .
$$

Clearly, Equation (40) does not agree with Eq. (38) unless $\alpha=$ 0 (a point on which we comment in Sect. 5).

Solving for $\bar{a}_{n}$ from Eq. (38), we find that

$$
\bar{a}_{n}(s)=-\frac{2(1+\tau s)}{n \pi\left[(1+\tau s) s+\lambda_{n}(1+\alpha s)\right]}=-\frac{2\left(\tau^{-1}+s\right)}{n \pi\left(s-s_{+}^{\bullet}\right)\left(s-s_{-}^{\bullet}\right)} .
$$

where $s_{ \pm}^{\bullet}=-\left[\left(1+\lambda_{n} \alpha\right) \pm \sqrt{\Delta_{n}}\right] /(2 \tau)$ with $\Delta_{n}:=\left(1+\lambda_{n} \alpha\right)^{2}-$ $4 \lambda_{n} \tau$. Using entries 11 and 18 from the Table of Laplace Transforms in Doetsch, 1974, pp. 317-321) for $\Delta_{n} \neq 0$ and entries 8 and 17 for $\Delta_{n}=0$, once again three cases can be distinguished and, finally, we arrive at the correct solution:

$u(y, t)=H(t)\left[(1-y)-\frac{2}{\pi} \sum_{n=1}^{\infty} \exp \left(-\frac{1+\lambda_{n} \alpha}{2 \tau} t\right) A_{n}(t) \frac{\sin (n \pi y)}{n}\right]$,

where

$$
A_{n}(t)= \begin{cases}\frac{1-\lambda_{n} \alpha}{\sqrt{\Delta_{n}}} \sinh \left(\frac{t}{2 \tau} \sqrt{\Delta_{n}}\right)+\cosh \left(\frac{t}{2 \tau} \sqrt{\Delta_{n}}\right), & \Delta_{n}>0, \\ \frac{1-\lambda_{n} \alpha}{2 \tau} t+1, & \Delta_{n}=0, \\ \frac{1-\lambda_{n} \alpha}{\sqrt{\left|\Delta_{n}\right|}} \sin \left(\frac{t}{2 \tau} \sqrt{\left|\Delta_{n}\right|}\right)+\cos \left(\frac{t}{2 \tau} \sqrt{\left|\Delta_{n}\right|}\right), & \Delta_{n}<0 .\end{cases}
$$


Clearly, this solution agrees exactly with the solution found in Sect. 3 using only the Laplace transform in time, specifically Eqs. (31) and (43) are identical. Thus, though it may appear the differences between the Jeffreys-fluid solutions in Sect. 2 and this section are minor (almost typographical in nature), these two Fourier series representations have fundamentally different properties as illustrated in Fig. 1.

One independent check on the exact solution for the start-up of plane Couette flow of a Jeffreys fluid found above is a direct numerical inversion of the Laplace-domain solutions (i.e., Eq. (22)). This can be achieved by a Riemann-sum approximation (Tzou, 1997, Sect. 2.5.1):

$$
u(y, t) \approx \frac{\mathrm{e}^{4.7}}{t}\left\{\frac{1}{2} \bar{u}\left(y, \frac{4.7}{t}\right)+\operatorname{Re}\left[\sum_{m=1}^{M}(-1)^{m} \bar{u}\left(y, \frac{4.7+\mathrm{i} m \pi}{t}\right)\right]\right\},
$$

where the value 4.7 has been fine-tuned numerically to ensure the accuracy of the approximation (Tzou, 1997, p. 41), and $M \gg 1$ is taken large enough to ensure that the sum has converged. Another independent check on the exact solution is a comparison to a numerical solution of IBVP (3) by the secondorder-accurate finite-difference scheme constructed by Christov 2010, Sect. 3.2) (using the same number of spatial and temporal grid points as therein). Both of these are shown as discrete symbols in Fig. 1 .

Since $\alpha$ and $\tau$ are dimensionless, for the purposes of Fig. 1 we take them to be $O(1)$ without loss of generality. In accordance with experimental observations (Toms and Strawbridge, 1953), we choose them such that $\alpha / \tau=0.5$.

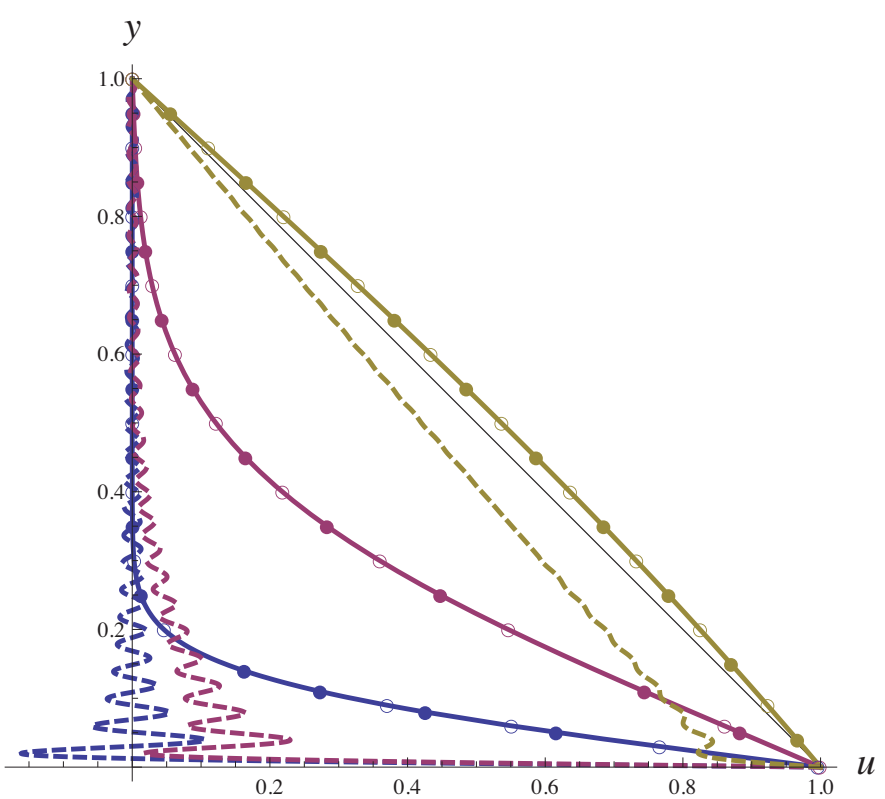

Figure 1: Approach to steady-state of the start-up flow of a Jeffreys' fluid (with $\tau=1$ and $\alpha=0.5$ ) between two parallel plates: 50 terms of Eqs. 15- 16 (bold dashed curves), 25 terms of Eqs. 42-43 (bold solid curves), Tzou's Riemann sum inversion from Eq. 44 (open symbols), numerical solution of IBVP 3 (filled symbols) and the steady-state from Eq. (4) (thin line). Darkest colors (blue online), intermediate dark colors (red online) and lightest colors (yellow online) correspond, respectively, to the solutions at $t=0.01,0.1$ and 1 .

\section{Discussion}

In this paper, we expounded on some subtle but critical aspects of the method of solution for transient/start-up problems in continuum mechanics. To the best of our knowledge, the erroneous approach of Sect. 2 was first introduced for the nonNewtonian case with $\tau=0$ by Rajagopal (1982). However, it did not become prominent in the literature until the paper by Bandelli and Rajagopal (1995) brought renewed interest to the topic (see also (Jordan, 2005)). Meanwhile, the incorrect solution to the start-up plane Couette flow of a Jeffreys (nowadays referred to as "Oldroyd-B") fluid appears to have been supplied by Hayat et al. (2001, 2004)5 As a result of many authors applying the erroneous technique from Sect. 2 to just about every variation of non-Newtonian rheology and boundary conditions, a vast literature of works presenting erroneous solutions now exists. For example, the incorrect solution for the start-up of plane Couette flow of a second-order fluid from (Rajagopal, 1982) has made its way into textbooks (Truesdell and Rajagopal, 2000, Sect. 7.2).

Here, we do not attempt to catalogue the various erroneous solutions, including those in which the lower plate velocity is set into oscillatory or otherwise time-dependent motion such that $u(0, t)=H(t) \mathfrak{F}(t)$, in which case matters are complicated by the fact that the mathematical error is self-canceling if $\mathfrak{F}(0)=0$. Detailed case studies by Christov (2010, 2011) and Christov and Jordan (2012) correct a related subset of the literature on non-Newtonian fluid mechanics in which erroneous solutions have been derived due to a (more mundane) mistake in applying the Fourier sine transform to a mixed derivative term (see also Christov and Jordan, 2009; Christov and Christov, 2010)). Furthermore, while the present work only deals with planar unidirectional flows, the same reasoning applies to startup problems in cylindrical domains wherein a fluid (respectively, a heat-conducting material) fills a cylinder or the gap between two cylinders, one or both of which is rotating and/or translating (respectively, one or both of which is differentially heated). Indeed, Bandelli and Rajagopal (1995, Sect. 6) commit the error from Sect.2 for a cylindrical geometry and $\tau=0$, but proceed to (mistakenly) claim that the earlier solution to the same problem by Ramkissoon (1985) is wrong. Nevertheless, the approach of Ramkissoon (1985) is not rigorous because the non-zero value of the imposed boundary condition at $t=0$ has been set to zero. A careful derivation (Christov, 2011) shows that despite this arbitrary and unjustified assumption, one can obtain the correct solution (for the wrong reason).

For $\alpha=0$ both the erroneous (Eqs. 15-16) and correct (Eqs. (42)-433) solutions reduce to the correct solution to the problem of start-up of plane Couette flow of a Maxwell fluid (Denn and Porteous, 1971) 6 For $\tau=\alpha=0$ the Newtonian

\footnotetext{
${ }^{5}$ It should be noted that Hayat et al. 2001, 2004) do not present their solution technique, instead they only state the final form of the solution. Since (Rajagopal, 1982) is cited in (Havat et al., 2001) and (Havat et al., 2001) is cited in (Havat et al., 2004), we are led to believe that Havat et al. (2001, 2004) arrived at the wrong solution by the textbook technique (as in Sect.2] herein).

${ }^{6}$ The solution given by Denn and Porteous (1971) is indeed the correct
} 
solution is recovered in all cases. Of course, reducing one's solution to a published result in some limit is not a proof that the former is correct, claiming so would be a logical fallacy. The only conclusion that can be drawn is that terms of the solution that cannot be verified to be correct are multiplied by the parameter that is being taken to zero. Nevertheless, the pernicious fact that the even erroneous solutions reduce to correct ones in such limits seems to have deterred proper verification of some solutions in the literature.

At the heart of the problem presented in this paper is the matter of the start-up jump 7 This is the phenomenon in which the solution to a start-up problem is discontinuous across the plane $t=0$. It appears to have been first identified by Tanner (Tanner, 1962) in solving the classical first problem of Stokes for the Jeffreys fluid. In general, it is thought that start-up jumps are due to the presence of mixed derivatives in the governing partial differential equation, as confirmed by a number of examples in the literature most recently for start-up flows of dipolar fluids (Jordan and Puri, 2002, Eq. (4.1)) and bubbly liquids (Jordan and Feuillade, 2006). However, the first exact solutions obtained by Ting (1963) for the second-order fluid $(\tau=0)$ and by Waters and King (1970) for the Jeffreys fluid did not exhibit start-up jumps, which appears to have led to the incorrect supposition in the literature that start-up jumps cannot occur for such non-Newtonian fluids (see, e.g., Truesdell and Rajagopal, 2000, p. 121) 8

In conclusion, it is the author's recommendation that the textbook description of start-up problems of fluid flow and of transient heat conduction be revised to include the approach from Sect. 4, so that the treatment is mathematically rigorous and applicable to non-classical problems.

\section{Acknowledgements}

This work was supported by NSF Grant DMS-1104047. The author is indebted to Dr. Pedro M. Jordan, Prof. Christo I. Christov, Prof. Howard A. Stone and Dr. Andrew J. Cihonski for con-

one because it can also be obtained by using only the Laplace transform in time (Jordan, 2011). There are, however, two typographical errors in (Denn and Porteous, 1971, Eq. (8)): (i) the pre-factors $1 / \lambda_{n}$ and $1 /\left(-\lambda_{n}\right)$ of the sin and sinh terms, respectively, should be $1 / \sqrt{\lambda_{n}}$ and $1 / \sqrt{-\lambda_{n}}$, where $\lambda_{n}$ in (Denn and Porteous, 1971) corresponds to $\Delta_{n}$ in the present work, and (ii) $T_{n}$ should be multiplied by -1 .

${ }^{7}$ It is important to note that this is distinct from the paradox identified by Sir Horace Lamb (Lamb, 1927), i.e., the discontinuity at $y=0$ of the function defined by the Fourier series $\sum_{n=1}^{\infty} \frac{1}{n} \sin (n \pi y)$, which is part of all the solutions presented herein in the limit as $t \rightarrow 0^{+}$(see also Batchelor, 1967, p. 191)). The way to reconcile both Lamb's paradox and the start-up jump phenomenon with our (perhaps unrealisitic) expectations of smoothness of solutions is to realize that the initial condition and the solution to the BVP for $t>0$ do not have to hold true at boundaries, as made explicit by the notation used to state IBVPs (1) and 3 herein. Indeed, the initial and boundary conditions for Stokes-type problems are incompatible, leading to a discontinuity even at $t=0$.

${ }^{8}$ Curiously, it appears that Eqs. (9.25) and (9.28) of Bandelli and Rajagopal (1995), which were obtained using only the Laplace transform in $t$, provide an alternative representation of the dimensional version of Eq. (3.4) of Jordan (2005), i.e., the correct solution to IBVP (3) (with $\tau=0$ ). However, on the grounds that Eqs. (9.25) and (9.28) of Bandelli and Rajagopal (1995) exhibit a start-up jump discontinuity, it was concluded by the latter authors that they do not represent the solution being sought. stant encouragement and many helpful remarks and discussions both on the subject matter and on the manuscript.

\section{References}

Bandelli, R., Rajagopal, K.R., 1995. Start-up flows of second grade fluids in domains with one finite dimension. Int. J. Non-linear Mech. 30, 817-839.

Bartels, R.C.F., Churchill, R.V., 1942. Resolution of boundary problems by the use of a generalized convolution. Bull. Am. Math. Soc. 48, 276-282. http://projecteuclid.org/euclid.bams/1183504258

Batchelor, G.K., 1967. An Introduction to Fluid Dynamics. Cambridge University Press, Cambridge.

Bird, R.B., Armstrong, R.C., Hassager, O., 1987. Dynamics of Polymeric Liquids. volume 1. John Wiley, New York. 2nd edition.

Bruus, H., 2008. Theoretical Microfluidics. volume 16 of Oxford Master Series in Condensed Matter Physics. Oxford University Press, Oxford.

Carslaw, H.S., Jaeger, J.C., 1959. Conduction of Heat in Solids. Clarendon Press, Oxford. 2nd edition.

Carslaw, H.S., Jaeger, J.C., 1963. Operational Methods in Applied Mathematics. Dover Publications, New York. 2nd edition.

Christov, C.I., 2008. On the evolution of localized wave packets governed by a dissipative wave equation. Wave Motion 45, 154-161.

Christov, C.I., Jordan, P.M., 2009. Comment on “Stokes' first problem for an Oldroyd-B fluid in a porous half space" [Phys. Fluids 17, 023101 (2005)]. Phys. Fluids 21, 069101.

Christov, I.C., 2010. Stokes' first problem for some non-Newtonian fluids: Results and mistakes. Mech. Res. Commun. 37, 717-723. arXiv:1009.4416

Christov, I.C., 2011. Comments on: "Energetic balance for the RayleighStokes problem of an Oldroyd-B fluid" [Nonlinear Anal. RWA 12 (2011) 1]. Nonlinear Anal. RWA 12, 3687-3690. arXiv:1107.2947

Christov, I.C., Christov, C.I., 2010. Comment on "On a class of exact solutions of the equations of motion of a second grade fluid" by C. Fetecău and J. Zierep (Acta Mech. 150, 135-138, 2001). Acta Mech. 215, 25-28. arXiv:1003.2188

Christov, I.C., Jordan, P.M., 2012. Comments on: "Starting solutions for some unsteady unidirectional flows of a second grade fluid" [Int. J. Eng. Sci. 43 (2005) 781]. Int. J. Eng. Sci. 51, 326-332. arXiv:1111.4464

Coleman, B.D., Noll, W., 1960. An approximation theorem for functionals, with applications in continuum mechanics. Arch. Rational Mech. Anal. 6, 355-370.

Courant, R., Hilbert, D., 1962. Methods of Mathematical Physics. volume II. John Wiley \& Sons, New York.

Crighton, D.G., 1998. Propagation of finite-amplitude waves in fluids, in: Crocker, M.J. (Ed.), Handbook of Acoustics. Wiley-Interscience, New York. chapter 17, pp. 187-202.

Denn, M.M., Porteous, K.C., 1971. Elastic effects in flow of viscoelastic liquids. Chem. Eng J. 2, 280-286.

Doetsch, G., 1974. Introduction to the Theory and Application of the Laplace Transformation. Springer-Verlag, Berlin/Heidelberg. Translated by W. Nader.

Duarte, A.S.R., Miranda, A.I.P., Oliveira, P.J., 2008. Numerical and analytical modeling of unsteady viscoelastic flows: The start-up and pulsating test case problems. J. Non-Newtonian Fluid Mech. 154, 153-169.

Duhamel, J.M.C., 1833. Sur la méthode générale relative au mouvement de la chaleur dans les corps solides plongés dans des milieux dont la température varie avec le temps. J. École Roy. Polytech. 22 (14), 20-77. http://gallica.bnf.fr/ark:/12148/bpt6k433678n/f21.image.langEN

Frohlich, H., Sack, R., 1946. Theory of the rheological properties of dispersions. Proc. R. Soc. Lond. A 185, 415-430.

Hayat, T., Khan, M., Ayub, M., 2004. Exact solutions of flow problems of an Oldroyd-B fluid. Appl. Math. Comput. 151, 105-119.

Hayat, T., Siddiqui, A., Asghar, S., 2001. Some simple flows of an Oldroyd-B fluid. Int. J. Eng. Sci. 39, 135-147.

Jeffreys, H., 1929. The Earth: Its Origin, History and Physical Constitution. Cambridge University Press, Cambridge. 2nd edition.

Jeffreys, H., 1932. On plasticity and creep in solids. Proc. R. Soc. Lond. A 138, 283-297.

Jordan, P.M., 2005. A note on start-up, plane Couette flow involving secondgrade fluids. Math. Prob. Eng. 2005, 539-545.

Jordan, P.M., 2011. private communication. 
Jordan, P.M., Feuillade, C., 2006. A note on Love's equation with damping. Proc. R. Soc. Lond. A 462, 2063-2076.

Jordan, P.M., Meyer, M.R., Puri, A., 2000. Causal implications of viscous damping in compressible fluid flows. Phys. Rev. E 62, 7918-7926.

Jordan, P.M., Puri, P., 2002. Exact solutions for the unsteady plane Couette flow of a dipolar fluid. Proc. R. Soc. Lond. A 458, 1245-1272.

Joseph, D.D., 1986. Historical perspectives on the elasticity of liquids. J. NonNewtonian Fluid Mech. 19, 237-249.

Lamb, H., 1927. A paradox in fluid motion. J. London Math. Soc. s1-2 (2), $109-112$.

Leal, L.G., 2007. Advanced Transport Phenomena: Fluid Mechanics and Convective Transport Processes. Cambridge University Press, New York.

Llewellin, E.W., Mader, H.M., Wilson, S.D.R., 2002. The rheology of a bubbly liquid. Proc. R. Soc. Lond. A 458, 987-1016.

Maxwell, J.C., 1867. On the dynamical theory of gases. Phil. Trans. R. Soc. Lond. 157, 49-88.

McArdle, C.R., Pritchard, D., Wilson, S.K., 2012. The Stokes boundary layer for a thixotropic or antithixotropic fluid. J. Non-Newtonian Fluid Mech. 185-186, 18-38.

Miller, H.R., Mattuck, A.P., 2010. Class 27 Lecture Notes: Application to ODEs. MIT OpenCourseWare 18.03 Differential Equations, As taught in: Spring 2010, http://ocw.mit.edu/courses/mathematics/18-03-differential-equations-spring-2010/lecture-notes/MIT18_03S10_c27.pdf

Nijenhuis, K., McKinley, G.H., Spiegelberg, S., Barnes, H.A., Aksel, N., Heymann, L., Odell, J.A., 2007. Non-Newtonian flows, in: Tropea, C., Yarin, A.L., Foss, J.F. (Eds.), Springer Handbook of Experimental Fluid Mechanics. Springer-Verlag, Berlin/Heidelberg. chapter 9, pp. 619-743.

Oldroyd, J.G., 1950. On the formulation of rheological equations of state. Proc. R. Soc. Lond. A 200, 523-541.

Pritchard, D., McArdle, C.R., Wilson, S.K., 2011. The Stokes boundary layer for a power-law fluid. J. Non-Newtonian Fluid Mech. 166, 745-753.

Rajagopal, K.R., 1982. A note on unsteady unidirectional flows of a nonNewtonian fluid. Int. J. Non-linear Mech. 17, 369-373.

Ramkissoon, H., 1985. Unsteady flow of a second-order fluid between concentric cylinders. Rheol. Acta 24, 623-626.

Sneddon, I.N., 2006. Elements of Partial Differential Equations. Dover Publications, New York.

Stokes, G.G., 1848. On a difficulty in the theory of sound. Phil. Mag. Ser. 3 33, 349-356.

Stokes, G.G., 1851. On the effect of the internal friction of fluids on the motion of pendulums. Trans. Cambridge Phil. Soc. 9 (Part II), 8-106. http://www.biodiversitylibrary.org/item/19878\#page/216/

Straughan, B., 2011. Heat Waves. volume 177 of Applied Mathematical Sciences. Springer, New York.

Tanner, R.I., 1962. Note on the Rayleigh problem for a visco-elastic fluid. Z. angew. Math. Phys. (ZAMP) 13, 573-580.

Ting, T.W., 1963. Certain non-steady flows of second-order fluids. Arch. Rational Mech. Anal. 14, 1-26.

Titchmarsh, E.C., 1962. Eigenfunction Expansions Associated with Secondorder Differential Equations, Part I. Clarendon Press, Oxford. 2nd edition.

Toll, J.S., 1956. Causality and the dispersion relation: Logical foundations. Phys. Rev. 104, 1760-1770.

Toms, B.A., Strawbridge, D.J., 1953. Elastic and viscous properties of dilute solutions of polymethyl methacrylate in organic liquids. Trans. Faraday Soc. 49, 1225-1232.

Truesdell, C., Rajagopal, K.R., 2000. An Introduction to the Mechanics of Fluids. Birkhäuser, Boston.

Tzou, D.Y., 1997. Macro- to Microscale Heat Transfer: The Lagging Behavior. Taylor \& Francis, Washington, DC.

Vasquez, P.A., Jin, Y., Vuong, K., Hill, D.B., Forest, M.G., 2013. A new twist on Stokes' second problem: Partial penetration of nonlinearity in sheared viscoelastic layers. J. Non-Newtonian Fluid Mech. 196, 36-50.

Waters, N.D., King, M.J., 1970. Unsteady flow of an elastico-viscous liquid. Rheol. Acta 9, 345-355. 